\title{
¿Resulta apropiado utilizar la guía telefónica como marco muestral \\ en sondeos?: \\ un estudio piloto \\ en la Comunidad navarra*
}

\author{
Vidal Díaz de Rada \\ Universidad Pública de Navarra \\ vidal@unavarra.es \\ Jaime Andréu Abela \\ Universidad de Granada \\ jandreu@ugr.es
}

RESUMEN

Cuando la población objetivo de un sondeo son colectivos especializados (médicos, abogados, etc.) es usual utilizar directorios profesionales de cada uno de éstos, pero ¿que ocurre cuando el objeto de estudio es la población general? En este trabajo se responde a esta pregunta, analizando hasta que punto es correcta la utilización de la guía telefónica como marco poblacional para encuestas personales (cara a cara), telefónicas y por correo. Con este fin exponemos los resultados de una investigación realizada con una encuesta postal, que posteriormente fue completada con una encuesta telefónica y otra personal. La hipótesis planteada postula que el principal problema de la utilización de la guía de teléfono como marco poblacional es la dificultad para conseguir una adecuada representatividad de la realidad objeto de estudio.

Palabras clave: Encuestas Postales, Encuestas Telefónicas, Marco Poblacional, No Cobertura.

\footnotetext{
* Una primera versión de este trabajo ha sido presentada en el III Congreso de Investigación Mediante Encuestas.
} 
Las primeras referencias en nuestro idioma a las encuestas telefónicas se realizaron en el ya clásico libro de Enrique Ortega Manual de Investigación Comercial, cuya primera edición (1981) ha cumplido ya los veinte años. Desde entonces la utilización de la encuesta telefónica en investigación comercial ha aumentado notablemente (cuadro 1), al tiempo que se ha producido un elevado incremento de la literatura especializada sobre el tema (Bosch y Torrente, 1993; Wert, 1994; Díaz de Rada, 2000, entre otros).

\section{CUADRO 1}

Número de encuestas realizadas en España según procedimiento de recogida de información (en porcentajes)

\begin{tabular}{lrrrrrrrrrr} 
& 2001 & 2000 & 1999 & 1998 & 1997 & 1996 & 1995 & 1994 & 1993 \\
\hline Encuesta personal & 36 & 38 & 56 & 46 & 66 & 64 & 56 & 64 & 65 \\
Encuesta telefónica & 48 & 41 & 30 & 44 & 28 & 29 & 36 & 32 & 25 \\
Encuesta postal & 8 & 6 & 5 & 7 & 6 & 7 & 8 & 4 & 9 \\
Otros & 8 & 15 & 9 & 3 & - & - & - & - & - \\
\cline { 1 - 5 } Total (miles) & 9.072 & 8.808 & 8.427 & 7.855 & 8.878 & 6.706 & 7.016 & 7.877 & 5.755
\end{tabular}

FUENTE:

Alós (1995a: 76); Alós (1995b: 76); Alós (1997: 74); Alós (1998: 62); Alós (1999: 75); Alós (2001: 74).

Sin embargo, y pese a esta situación, llama la atención la escasa investigación realizada en nuestro país sobre la adecuación de los marcos muestrales utilizados en encuestas telefónicas. Aunque un importante número de encuestas telefónicas utilizan sistemas de números aleatorios (RDD) ${ }^{1}$ para la selección de los números de teléfono de las personas que serán entrevistadas, la mayor parte de las encuestas telefónicas realizadas en nuestro país emplean directorios de teléfonos publicados por los proveedores del servicio, siendo el más utilizado la guía de teléfonos de Telefónica. Investigaciones realizadas en otros países han demostrado los problemas de cobertura producidos por la utilización de estas guías: en el Reino Unido, diversas investigaciones realizadas a principios de la década de los noventa del siglo xx (Foreman y Collins, 1991; Thomas y Purdon, 1994: 1) estiman que el $25 \%$ de las personas que tienen teléfono no están incluidas en los listines telefónicos; porcentaje que aumenta hasta el 36\% a mediados de la década (Noble et al., 1998). En Estados Unidos, Lavrakas (1993: 34) señala que la mitad de la población de algunas ciudades

1 Diversas investigaciones han señalado que esta estrategia produce una gran cantidad de números de teléfono que no son hogares, así como números que no existen. Para ello, Traugott et al. (1987: 524) proponen utilizar este método conjuntamente con la selección aleatoria de la guía telefónica. 
como Chicago no está en la guía, porcentaje que se reduce al $25-35 \%$ en la población que reside en los alrededores de Chicago ${ }^{2}$.

En nuestro país, donde en el año 2001 se llevaron a cabo más de cuatro millones de encuestas telefónicas $^{3}$, no se han realizado investigaciones para conocer la adecuación de la guía de teléfonos de la empresa Telefónica como marco muestral, hecho que puede generar problemas en la selección y definición de la población objeto de estudio.

\section{INTRODUCCIÓN}

Uno de los momentos más importantes en la investigación mediante encuesta es la selección y definición de la población objeto de estudio, en la medida que limita el objeto de la investigación a un determinado entorno sociogeográfico. En la definición de la población relevante para la investigación, Groves (1989: 82) distingue tres tipos de poblaciones: población de inferencia, población objetivo y población medida. La población de inferencia está formada por el conjunto de personas objeto de estudio. El segundo tipo de población recibe el nombre de población objetivo, y es definida como un número determinado de personas que van a ser estudiadas en un momento dado. La diferencia entre ambas es realizada por el investigador (Groves, 1989: 82), de modo que la población objetivo está referida a las personas que viven en hogares principales dentro del área estudiada en un determinado período de tiempo. Es decir, es la población de inferencia menos las personas en instituciones, en bases militares, en lugares remotos del país, y aquellas sin domicilio (Groves, 1989: 99). La población objetivo está recogida en el marco poblacional, documento de donde se seleccionan las personas objeto del estudio. Por último, la población medi$d a$, referida al conjunto de personas que responden al cuestionario al ser seleccionadas en la muestra.

Del párrafo anterior se desprende que el marco poblacional no es otra cosa que un documento donde se recoge la población objetivo, y adquiere un papel muy relevante en el diseño de la investigación porque de este documento será seleccionada la población a entrevistar. Normalmente, el marco poblacional es una lista de los individuos que viven en un determinado entorno, un listado de hogares con direcciones, números de teléfono, etc.

\footnotetext{
2 Una explicación exhaustiva de los métodos más utilizados para resolver este problema presenta Lepkowski (1988: 81-98), con una aplicación a la realidad de los Estados Unidos. En el contexto europeo, Foreman y Collins (1991) han comprobado la escasa eficacia de estos métodos para resolver el problema de los números no listados en el Reino Unido. Collins (1999: 2-3) presenta ejemplos de otros contextos donde estos métodos no proporcionan resultados adecuados.

3 Concretamente, 4.354.560. Cifra obtenida multiplicando las 9.072 .000 encuestas realizadas en el año 2001 por 0,48, que es el porcentaje de encuestas realizadas a través del teléfono.
} 
Cuando no se cuenta con un listado de los individuos o de los hogares que viven en un determinado espacio, las entrevistas personales emplean como marco poblacional mapas sobre áreas de la ciudad, mientras que las entrevistas telefónicas utilizan sistemas de números aleatorios (RDD). Ambas estrategias han mostrado excelentes resultados en las entrevistas personales y telefónicas, pero no pueden utilizarse para las encuestas postales puesto que éstas precisan de un marco más completo.

Cuando la población objetivo en un sondeo son colectivos especializados (médicos, abogados, etc.) es usual la utilización de directorios profesionales de cada uno de éstos, pero ¿qué ocurre cuando el objeto de estudio es la población general? En este trabajo se responde a esta pregunta, analizando hasta qué punto es correcta la utilización de la guía telefónica como marco poblacional para encuestas personales (cara a cara), telefónicas y por correo. Para ello se presentan los resultados de una investigación, realizada en Navarra en la primavera de 1998, utilizando una encuesta postal que posteriormente fue completada con una encuesta telefónica y otra personal. La hipótesis de trabajo es que el principal problema de la utilización de la guía de teléfonos como marco poblacional es la dificultad para conseguir una adecuada representatividad de la realidad objeto de estudio.

\section{DISEÑO DE UNA MUESTRA UTILIZANDO LA GUÍA DE TELÉFONOS}

Antes de proceder con la decisión sobre el tamaño muestral es necesario llevar a cabo la selección de un documento que recoja de forma adecuada la población a estudiar. Numerosos expertos han demostrado que la guía de teléfonos puede ser utilizada como marco para población general en encuestas postales porque está disponible y tiene bajo coste (entre otros, Kviz, 1984: 801; Keeter, 1995). No obstante, en su elección debe considerarse la presencia de diversos problemas, entre los que Dillman (1978: 42-44) señala:

a) Es posible que determinados segmentos de la población general no dispongan de los atributos necesarios para formar parte de la muestra. Si se emplean guías de teléfonos, por ejemplo, se produce un error de cobertura al no considerar la población sin teléfono. En 1998 , el $83 \%$ de los hogares españoles tienen teléfono en su hogar, porcentaje que llega al 95\% en Navarra (Centro de Investigaciones Sociológicas, 1998; Díaz de Rada, 2004).

b) Muchas de las listas utilizadas no están actualizadas, de modo que no son consideradas las personas que acaban de instalar un teléfono, al tiempo que son incluidas otras personas que ya se han dado de baja. Es posible que estos dos tipos de personas sean colectivos con unas características concretas y totalmente diferentes del resto de la población, de modo que la no inclusión de estas personas alterará significativamente la calidad de la 
muestra recogida. Así, más que el porcentaje de población listada, interesa conocer si existen diferencias entre los que aparecen y los que son excluidos de los listados telefónicos.

Aunque algunas investigaciones realizadas en otros contextos estiman que uno de cada tres titulares del teléfono no está incluido en las guías telefónicas (Foreman y Collins, 1991; Lavrakas, 1993: 34; Thomas y Purdon, 1994: 1, y Noble et al., 1998), otras investigaciones señalan que tan sólo el $4 \%$ de la población está excluida de los listados telefónicos y, lo que es más importante, que apenas existen diferencias entre la población listada y no listada (Brick et al., 1995: 218).

Esta situación puede estar provocada por errores en la inscripción de los datos, así como por el deseo de mantener la privacidad (y por ello hay personas que manifiestan sus deseos de no aparecer en la guía de teléfonos). A juicio de Wert (1994: 181), éste es un fenómeno muy variable según el país: el 15\% de la población residente en Estados Unidos no figura en las listas telefónicas, mientras que en España es un comportamiento muy minoritario. Bosch y Torrente estiman que en España tan sólo 100.000 personas no aparecen en las guías telefónicas, que de un total de 12 millones de teléfonos en funcionamiento representan apenas un 0,83\% (Bosch y Torrente, 1993: 92).

En 1998 existía en España una única compañía de teléfono, lo que garantiza que prácticamente todas las personas con teléfono estén recogidas en sus directorios ${ }^{4}$. Además, el cambio de domicilio en la sociedad española es muy bajo, aspecto que origina que existan escasas variaciones en las sucesivas ediciones de las guías telefónicas (que actualmente se renuevan cada dos años).

c) Estas listas, normalmente, incluyen el nombre del propietario de la vivienda, pero no el de las personas que viven en ella, lo que plantea dificultades para entrevistar a otros miembros de la vivienda.

d) Puede haber nombres incluidos más de una vez. Una persona puede tener más de un teléfono, aumentando con ello su posibilidad de selección muestral ${ }^{5}$. Nos referimos no sólo a los teléfonos de segundas viviendas ${ }^{6}$, sino fundamentalmente a las situaciones donde el número de teléfono corresponde a un negocio, una empresa, un bar, etc.

\footnotetext{
4 Debe tenerse en cuenta que en el año 1998 esta compañía no ofrecía la posibilidad de quedar excluido de las guías telefónicas, salvo contadas excepciones. A partir del año 1999 la compañía pregunta al usuario, en el momento de contratar la línea, si desea ser incluido en los listines telefónicos.

5 Un hogar puede tener más de un número de teléfono, situación no muy común en nuestro país.

6 De los 20.946.554 viviendas familiares existentes en nuestro país en el año 2001 (según el Censo de Población y Viviendas), el 16\% eran viviendas secundarias, usadas sólo en vacaciones, fines de semana, etc. (INE, 2004: 43).
} 
e) Dificultad de acceso a ciertas listas de personas por su confidencialidad (Censo de Población, por ejemplo).

Una vez considerados estos problemas se utilizó como marco poblacional la guía telefónica disponible en la primavera de 1998, realizada en el año 1996. Esta elección precisa una reflexión sobre los problemas enunciados unas líneas más arriba:

a) En primer lugar, la tasa de cobertura del teléfono es superior al 95\%, con lo cual menos del $5 \%$ de la población no está cubierta en esta investigación.

b) Respecto al segundo punto, la actualización de estas listas, veremos más adelante el número de direcciones no operativas por utilizar en 1998 una guía de teléfonos editada dos años antes (en 1996).

c) Aunque en la guía de teléfonos normalmente aparece el nombre del cabeza de familia, en la carta de presentación que acompaña al cuestionario se indicó que éste fuera respondido por una mujer adulta, y en la otra mitad se pedía que fuera respondido por la mayor de sus hijas. Si no había mujeres en el hogar se pedía que fuera respondido por un hombre o por el mayor de sus hijos (Hagen y Collier, 1983).

d) Respecto al número de nombres incluidos más de una vez, se trata de uno de los aspectos más problemáticos por las dificultades para conocer el número de personas incluidas más de una vez.

e) El último aspecto, relacionado con la confidencialidad del marco poblacional, no presenta problemas al emplear guías telefónicas, por la accesibilidad de éstas.

Relacionado con este aspecto, consideramos adecuado detenernos en un problema derivado de la utilización de la guía de teléfonos. En el ámbito anglosajón — que es donde más se ha desarrollado el método de la encuesta-, la mayor parte de la población reside en viviendas individuales identificadas con una calle y un número, mientras que en España la mayor parte de la población urbana vive en torres de pisos, que suelen tener entre 8 y 10 alturas y un promedio de 4 viviendas en cada planta. ¿Qué interés tiene esta configuración de la ciudad en el tema que nos ocupa? Este aspecto es importante porque la guía de teléfonos muestra el nombre del titular del teléfono, la calle donde vive y el número de la vivienda, pero no dice nada del piso donde reside. Es decir, es el personal de correos el que busca la referencia de esa persona entre todos los que viven en este edificio: así, en un edificio de 8 plantas y de 4 viviendas por planta, el cartero buscará el nombre del destinatario entre las 32 viviendas. 
La mayor parte de las viviendas tienen cerca de la puerta el buzón con el nombre de las personas que viven en cada vivienda, hecho que facilita la tarea de localización del entrevistado. Pero algunos edificios no tienen buzones con nombre, aspecto que dificulta que el personal de correos deposite la carta en su destino. Desde nuestro punto de vista, uno de los grandes problemas que tiene la utilización de guías de teléfonos en ciudades es la enorme dificultad en la localización de un hogar, teniendo una gran dependencia del servicio de correos.

En la investigación realizada en Navarra dividimos el marco poblacional según la sección censal a la que pertenece cada hogar: Pamplona está dividida en 7 distritos y 127 secciones censales. De este modo, la guía de teléfonos fue dividida en 133 secciones censales: 127 en Pamplona, 2 en Tudela y 1 en el resto de municipios.

El tamaño muestral para conocer adecuadamente la realidad navarra se estableció en 502 entrevistas, realizando 252 en un hábitat urbano (Pamplona, 166.279 habitantes), 50 en un municipio semiurbano (Tudela, 26.857 habitantes), otras 50 en un municipio mayor de 10.000 habitantes (Estella, 12.552 habitantes) y el resto en 3 municipios menores de 10.000 habitantes (Caparroso, 2.331 habitantes; Corella, 6.329, y Los Arcos, 1.346 habitantes). Este tamaño muestral proporciona un error de muestreo del 4,28\% para $p=0,50$ y con un nivel de confianza del $95 \%$. Considerando que han sido respondidos 270 cuestionarios $^{7}$, el error real alcanza el 5,84\%, con los mismos parámetros de proporcionalidad y nivel de confianza.

El cuestionario utilizado está formado por 15 preguntas sobre temas de consumo y cuatro preguntas de identificación (ver una copia del mismo en el anexo 1). Con el fin de contextualizar los resultados obtenidos en un entorno más amplio se tomaron preguntas de otras investigaciones: concretamente, la pregunta 5, la 6 y la 13 han sido utilizadas por De Miguel (1994: 376, 379 y 608) en sus estudios sobre la realidad española; mientras que la pregunta 11 forma parte de las encuestas de valores realizadas en el País Vasco (Elzo, 1992: 394), y la pregunta 12 del estudio La juventud liberta, dirigido por Ruiz Olabuénaga (1998: 331).

Respecto a la elaboración del cuestionario, tras la selección de las variables pertinentes para el objetivo de la investigación seguimos las recomendaciones de Dillman (1978: 79160 y 2000: 32-148) para hacer más fácil la respuesta del cuestionario. Para Dillman, es esencial que el cuestionario sea fácil de completar, dando una gran importancia a que sea

\footnotetext{
7 En los próximos apartados se explican las causas e implicaciones de esta tasa de respuesta; de la diferencia entre la mues-
} tra teórica (502) y la realmente conseguida. 
atractivo y cómodo en su configuración. En la elaboración del cuestionario se han utilizado las siguientes pautas:

- No se ha realizado ninguna pregunta en la primera página, utilizando este espacio para colocar un título interesante y un mapa del área de estudio. Se ha aprovechado también esta primera página para realizar una breve explicación sobre cómo rellenar el cuestionario. Tampoco se han colocado preguntas en la última página, dejando este espacio para recoger comentarios adicionales de los entrevistados.

- Las preguntas se han ordenado temáticamente, colocando al principio las más interesantes para el entrevistado.

- Se ha prestado un interés fundamental a la primera pregunta, buscando que sea interesante y fácil de responder. Hay que tener presente que la probabilidad de que se responda el resto del cuestionario depende en gran medida de esta primera pregunta.

- En referencia a los aspectos visuales, se han utilizado letras minúsculas en las preguntas y mayúsculas para las respuestas; las respuestas aparecen en formato vertical; se ha evitado que una pregunta aparezca «partida» en más de una página; y todas las páginas del cuestionario están escritas en su totalidad (sin espacios blancos en la parte inferior).

- Por último, y para evitar el parecido con cualquier tipo de publicidad postal, el cuestionario se imprimió sobre papel blanco y fue enviado empleando tarifa postal de primera clase. En el anexo 2 se adjunta la carta de presentación que acompañó al cuestionario.

\section{RESULTADOS OBTENIDOS}

Dividiremos este apartado en dos partes. En la primera se analizan el número de cuestionarios recibidos por sus destinatarios y cuántos de éstos han sido devueltos; mientras que en la segunda se comparan los rasgos sociodemográficos de las personas entrevistadas con la actualización del Censo de Población realizada en 1996 mediante el Padrón (Gobierno de Navarra, 1997 y 1998).

\section{A. Cuestionarios recibidos y calidad del marco poblacional}

Comenzaremos analizando la calidad del marco poblacional utilizado, considerando el número de cuestionarios que han llegado a su destino y cuántos han sido cumplimentados y devueltos (tasa de respuesta). En la cuarta línea de la tabla 1 se exponen diferentes pro- 
blemas generados por el marco utilizado, una guía telefónica con una antigüedad de dos años. Como puede apreciarse, un $12 \%$ de los cuestionarios no han llegado a su destino, o han llegado a un destino que no se correspondía con la información aportada con la guía de teléfonos. Analicemos en detalle cada uno de estos elementos:

- El problema más grave es el elevado número de cartas devueltas por direcciones incorrectas, problema que afecta a un $7,6 \%$ de la muestra.

- Dos semanas más tarde del envío del cuestionario realizamos una llamada de teléfono donde se comprobó que un $3,2 \%$ de los destinatarios no habían recibido el cuestionario enviado (y, sin embargo, tampoco había sido devuelto). Se ha utilizado esta estrategia animados por los excelentes resultados obtenidos por Nederhof en los Píses Bajos (1988). Nederhof descubrió que un $20 \%$ de la muestra no había recibido el cuestionario, porcentaje notablemente superior que el obtenido en esta investigación.

- Por último, en el 1,2\% $(0,4+0,8)$ de las viviendas seleccionadas se detectaron errores, producidos porque el residente actual no se correspondía con el nombre que aparecía en la guía de teléfonos. En varios casos, las viviendas seleccionadas tenían un uso distinto al de vivienda principal.

Estos tres factores suponen una reducción de la muestra teórica de 502 entrevistas a 442 (502 - \{38+16+2+4\}). Los 270 cuestionarios respondidos suponen, de los 442 enviados y recibidos por los destinatarios, una tasa de respuesta del $61 \%$.

TABLA

Cuestionarios recibidos y calidad del marco poblacional

\begin{tabular}{lrr} 
& N & Porcentaje \\
\hline Respuesta & 270 & 53,8 \\
Rechazo (en llamada telefónica) & 44 & 8,8 \\
No responde & 128 & 25,5 \\
Problemas con el marco utilizado & 60 & 12,0 \\
- Devolución & 38 & 7,6 \\
- No han recibido cuestionario & 16 & 3,2 \\
- Detectados errores en las llamadas telefónicas & & 0,4 \\
$\quad$ Reside otra persona & 2 & 0,8 \\
$\quad$ No es un hogar & 4 & 100,0
\end{tabular}


La información contenida en la tabla 2 matiza algunos de estos comentarios al mostrar la incidencia de cada uno de estos problemas en la muestra urbana y en la rural. Así, el número de cartas devueltas por direcciones incorrectas es mayor en el ámbito urbano, sin duda por el tipo de vivienda en el que se reside: torres de pisos que requieren un mayor esfuerzo por parte del servicio de correos. También el número de errores detectados en las Ilamadas telefónicas es superior en la ciudad, mientras que los pueblos destacan por su elevado número de personas que no recuerdan la recepción del cuestionario.

TABLA 2

Cuestionarios recibidos y calidad del marco poblacional en hábitat rural y urbano (en porcentajes) Cuestionarios recibidos

\begin{tabular}{lrr} 
& Pamplona & Pueblos \\
\hline Respuesta & 55,5 & 52,0 \\
Rechazo (en encuesta telefónica) & 9,5 & 8,0 \\
No responde & 19,9 & 31,2 \\
Problemas con el marco & 15,1 & 8,8 \\
\hline Total $(\mathrm{n})$ & 252 & 250
\end{tabular}

Problemas del marco poblacional

(Base $=60$ entrevistas que presentan problemas en el marco poblacional)

\begin{tabular}{lrr} 
& Pamplona & Pueblos \\
\hline Devolución (dirección incorrecta) & 85,0 & 20,0 \\
No han recibido cuestionario & 0,0 & 80,0 \\
Detectados errores en las llamadas telefónicas & & \\
$\quad$ Reside otra persona & 5,0 & 0,0 \\
No es un hogar & 10,0 & 0,0 \\
\hline Total $(\mathrm{n})$ & 40 & 20
\end{tabular}

El elevado número de personas que no han recibido el cuestionario, unido a la baja tasa de respuesta conseguida en el ámbito rural, nos llevan a analizar investigaciones sobre las características de los que no responden a encuestas, llegando a la conclusión que existe un acuerdo unánime en las características de las personas que más responden a encuestas: nivel educativo alto, alto status socioeconómico, más mujeres que hombres, solteros, jóvenes, residen en hábitat rural, etc. (McDaniel et al., 1987; DeMaio, 1980: 228-29). Si prácticamente todas las investigaciones destacan que la cooperación es mayor en hábitat rural, ¿cuál es la razón de esta baja cooperación en los datos obtenidos por nuestra investiga- 
ción? Tras un cuidadoso análisis de los factores que influyen en la no respuesta, una investigación cualitativa utilizando entrevistas en profundidad con líderes sociales de estos municipios nos permitió conocer que la baja respuesta estaba provocada por el gran número de viviendas de vacaciones (segundas residencias) que existen en algunos de estos municipios. La gran mayoría de estas viviendas se ocupan en verano, así que durante la realización del trabajo de campo (primavera) éstas estaban vacías. Sin duda, el correo almacenado se tira cuando se vuelve a ese lugar de vacaciones, razón por la que no recuerdan haber recibido el cuestionario.

\section{B. Representatividad y sesgo en los resultados obtenidos}

La forma más sencilla para conocer si los datos recogidos representan adecuadamente la población objeto de estudio se basa en una comparación entre éstos y el marco poblacional (Lessler y Kasbeek, 1992: 170). Si las diferencias encontradas se encuentran dentro del error muestral concluiremos afirmando que el error del marco es despreciable, basados en las semejanzas existentes entre ambos colectivos.

Uno de los problemas es que con este proceso estamos cuantificando no sólo los errores producidos por el marco muestral, sino también los generados por otros motivos como el error de medida, errores voluntarios e involuntarios producidos por el entrevistado, estrategia de selección de éste, fiabilidad de las preguntas, no respuesta total y parcial, etc. Con el fin de reducir la no respuesta total, y sin olvidar que el objetivo es considerar la adecuación de la guía de teléfonos como marco muestral, los que no respondieron fueron posteriormente entrevistados por teléfono, y aquellos que no fueron localizados - o que declinaron responder a la encuesta telefónica- fueron visitados por un entrevistador. Así, de las 172 personas que no respondieron a la encuesta postal fueron entrevistadas con el teléfono o mediante entrevista personal 106, lo que implica una tasa de respuesta total del $85 \%(\{270+106\} / 442)$. Considerando este nuevo tamaño muestral, el error de muestreo se reduce hasta el $5,15 \%$ para $p=0,50$ y con un nivel de confianza del 95\%.

Otro de los factores que dificultan tremendamente una correcta utilización de esta estrategia (comparación con el marco poblacional) es la localización de documentos que reflejen con exactitud las características de la población objeto de estudio, la necesaria actualización de esta información y los escasos datos que suelen incluir estos documentos. Respecto a este último punto, en determinadas ocasiones tan sólo será posible la comparación de unas pocas variables, generalmente las sociodemográficas, que no permiten un conocimiento adecuado de la magnitud del problema. 
Pese a estos problemas, se ha realizado una comparación entre los datos obtenidos por la encuesta y el marco poblacional elegido, el Padrón de 1996, analizando concretamente cuatro variables: nivel de estudios, tamaño de la familia, edad y sexo de los entrevistados:

- Respecto al nivel de estudios, numerosas investigaciones han señalado que las personas que más responden encuestas presentan estudios elevados, aspecto que produce una sobreestimación de los estratos con altos niveles de estudios. Como puede apreciarse en la primera parte de la tabla 3, la comparación entre el Padrón y los datos obtenidos por la encuesta da cuenta de esta tendencia, aunque las diferencias son escasas y en ningún caso llegan a ser significativas.

- En cuanto al tamaño del hogar, la segunda parte de la tabla 3 desvela que la investigación realizada infrarrepresenta los hogares en los que vive una persona y aquellos con más de cinco miembros. En este caso las diferencias comienzan a ser importantes, fundamentalmente por la infraestimación de los hogares más numerosos ${ }^{8}$.

- El análisis de la edad del entrevistado muestra que los más jóvenes son los que menos han respondido a la encuesta, y dentro de éstos destaca el colectivo entre 25 y 34 años por su gran diferencia con el Padrón, diferencia que es significativa al 99\%. Por otro lado, la encuesta realizada sobreestima la edad de los entrevistados con más años, fundamentalmente las personas entre 35 y 54 años, así como la población de más edad.

- El sexo no desvela diferencias importantes. Hay una ligera sobrerrepresentación de las mujeres, originada probablemente por la elevada edad de los entrevistados, puesto que el $24,5 \%$ de la muestra son mayores de 65 años, porcentaje que en la población llega al $14,5 \%$. En la explicación de este hecho debemos recordar que a medida que aumenta la edad de la población, la tasa de feminidad aumenta ${ }^{9}$. De hecho, si se ponderan las entrevistas de los mayores de 65 años (con el fin de que representen el 14\% de la muestra) desaparecen las diferencias en el sexo.

Concluimos este apartado señalando que las encuestas basadas en guías telefónicas consiguen una aceptable representación de la población respecto a su nivel de estudios, plan-

\footnotetext{
8 Uno de los métodos más utilizados para la selección de los entrevistados dentro del hogar, el propuesto por Kish en 1949, también presenta problemas para representar a los hogares de más tamaño (Kish, 1949: 384-386).

9 En el año 1996 las mujeres representaban el 52,3\% del grupo de edad entre 65 a 69 años. Este porcentaje aumenta al $54,6 \%$ entre el grupo entre 70 y 74 años, al $59,6 \%$ entre los 75 y 79 años, al $64,1 \%$ entre los 80 y 84 años, y al $68,5 \%$ entre los mayores de 84 años. Sintetizando, del grupo entre 65 y 74 años, el $53 \%$ son mujeres.
} 
teando algunos problemas a la hora de recoger información de los hogares más numerosos. El problema más importante aparece a la hora de conseguir la cooperación de las personas más jóvenes, con desviaciones que llegan al 10,6\% en el grupo entre 25 y 34 años.

TABLA 3

Características de los que responden en relación a la población total

Padrón 1996

A) Nivel de estudios

Menos primarios

Primarios-Primer grado

Segundo grado

Escuela universitaria

Superiores
Muestra

Diferencia (1)
Total

B) Tamaño del hogar

1 miembro

2 miembros

3 miembros

4 miembros

5 miembros y más

Total

C) Edad

18-24 años

25-34 años

35-44 años

45-54 años

55-64 años

65-74 años

Total

D) Sexo

Hombres

Mujeres

Total

356.578

48,9

51,1

11,2

22,5

20,1

17,6

14,5

13,9

356.578

$-3,4$

$+3,4$

* $<0,05$.

** $<0,01$.

(1) Valores negativos: colectivos infrarrepresentados por la encuesta.

FUENTE de los valores poblacionales: Gobierno de Navarra (1997 y 1998).
$-2,2$

$+2,6$

$+3,8$

$+3,5$

$-7,2^{*}$
376
45,5

54,5

7,8
11,7

22,1

19,6

14,3

24,5

376

370
$-3,4$

$-10,8^{\text {** }}$

$+2,0$

$+2,0$

$-0,2$

$+10,6^{\text {** }}$ 


\section{CONCLUSIONES}

El objetivo de este trabajo es conocer si es apropiado utilizar la guía telefónica como marco poblacional en encuestas, adoptando como hipótesis de trabajo que el principal problema de la utilización de la guía de teléfonos como marco poblacional es la dificultad para conseguir una adecuada representatividad de la realidad objeto de estudio. En las tablas 1 y 2 se ha analizado la viabilidad de utilizar una guía de teléfonos que tiene dos años de antigüedad. Aunque un $12 \%$ de la muestra seleccionada no ha recibido el cuestionario, en un $7,6 \%$ de los casos se ha producido por enviarlo a direcciones incorrectas, y por ello fue devuelto al investigador. Una llamada telefónica realizada unos días después desveló que un 3,2\% de la muestra no recordaba haber recibido el cuestionario, aspecto que puede ser imputable a la dificultad de localizar un determinado hogar en un edificio donde viven muchas personas, como señalábamos en el primer apartado.

Los problemas con el marco poblacional se reducen notablemente en el mundo rural, puesto que de los 60 cuestionarios con problemas, tan sólo 20 fueron enviados a municipios pequeños. Además, los problemas de este ámbito son distintos ya que se han producido muy pocas devoluciones del cuestionario por direcciones incorrectas.

En la tabla 3 se han comparado las características de la población (según el Padrón de 1996) con las características de las personas entrevistadas. Apenas existen diferencias entre el nivel de estudios de los entrevistados y el Padrón. En relación al tamaño de la familia, los hogares formados por tres personas aparecen sobrerrepresentados en un 3,8\%, mientras que los hogares más numerosos están infrarrepresentados. Sin ninguna duda, es la edad la variable que presenta diferencias más importantes, fundamentalmente por la infrarrepresentación de los colectivos menores de 34 años. Dos consideraciones podemos hacer de esta situación, la primera relativa a la calidad del marco poblacional, y la siguiente referida a la posibilidad que algunas de estas personas hayan decidido no responder:

1) En relación al primer aspecto debemos tener en cuenta que las personas más jóvenes son las que experimentan mayores cambios de residencia, de modo que es muy posible que la recogida padronal realizada en 1996 refleje muy poco la estructura de estos hogares en la primavera de 1998, que es cuando se realizó esta investigación.

La guía de teléfonos de 1996 —utilizada en 1998- puede presentar un importante error de cobertura que afecta fundamentalmente a este colectivo, puesto que es posible que estas personas no vivan ya en los hogares seleccionados por haberse emancipado. Se trata de colectivos con gran movilidad y, de hecho, el Informe Juventud en Navarra 2000 (López Blasco y otros, 2002: 67-69) detecta que a partir de los 26 años aumenta notablemente el 
número de jóvenes emancipados ${ }^{10}$, lo que implica que en la primavera de 1998 (cuando se realizó la investigación) había menos jóvenes viviendo en los hogares navarros que los mostrados por el Padrón de 1996.

2) Respecto a la no respuesta de estas personas, uno de los mayores especialistas mundiales en el tema de la no respuesta señala que los más jóvenes son los que más responden a entrevistas personales y telefónicas, llegando a plantear que la edad correlaciona negativamente con la probabilidad de responder. Más problemas plantea la posibilidad de contactar con estas personas, puesto que en determinadas edades el tiempo que se está en casa es muy poco (Goyder, 1987: 85).

A modo de conclusión y resumen final refutamos la hipótesis planteada, puesto que no se producen grandes desajustes en la población objetivo cuando se utilizan guías telefónicas como marco poblacional (Sudman, 1973), aunque estos desajustes son menores en el ámbito rural que en el urbano. Si consideramos que la guía telefónica utilizada tiene una antigüedad de dos años, y en España cada dos años se cambian las guías de teléfono, utilizar una guía con una antigüedad menor (un año, o año y medio) permitirá obtener unos mejores resultados.

Pese a estos resultados, en el momento actual nos enfrentamos a un nuevo problema con la aparición de los teléfonos móviles. El enorme incremento de los teléfonos móviles en los últimos años, y la no aparición de estos números en las guías telefónicas, pueden plantear problemas de representatividad en las muestras (De Leeuw et al., 2002; Kuusela y Simpanen, 2002, y Pasadas del Amo et al., 2004: 4). Sin embargo, en el momento presente este problema no es muy importante en España, puesto que el elevado coste de las llamadas con estos teléfonos ha limitado que sustituyan a los teléfonos fijos. Por este motivo, nos encontramos en una situación en la que conviven —en una misma vivienda - un teléfono fijo con varios móviles. Este hecho, qué duda cabe, va a experimentar una gran transformación dentro de poco tiempo.

A pocas semanas de la publicación de este trabajo hemos conocido una investigación que proporciona datos de la incidencia de este problema en Andalucía. Utilizando datos del año 2004, Pasadas del Amo et al. (2004) señalan que un 23\% de los hogares andaluces disponen únicamente de teléfono móvil; y que estos hogares se caracterizan por ser de reciente creación, estar formados por personas jóvenes en situaciones laborables inestables o en período de transición (estudiantes en pisos compartidos, trabajadores en prácticas, etc). Cuando se analizan específicamente los hogares formados por personas jóvenes en situación de independencia se produce una gran sustitución del teléfono fijo por el móvil: «casi

10 Según datos del Instituto de Estadística de Navarra (2004), entre 1996 y 1998 se realizaron en Navarra 16.008 matrimonios. De éstos, en 11.855 (el 74\%) los contrayentes tenían entre 25 y 34 años; en 1.747 (11\%) los contrayentes tenían entre 20 y 24 años. 
uno de cada dos hogares formados por jóvenes menores de 35 años carece de teléfono fijo» (Pasadas del Amo et al., 2004: 10).

\section{REFERENCIAS}

ALÓS, J. S. (1995a): «Industria de los estudios de mercado en España 1993», Investigación y Marketing, vol. 47, pp. 74-77.

- (1995b): «Industria de los estudios de mercado en España 1993», Investigación y Marketing, vol. 48, pp. 74-77.

- (1997): «Industria de los estudios de mercado en España 1996», Investigación y Marketing, vol. 56, pp. 73-74.

- (1998): «Industria de los estudios de mercado en España 1997», Investigación y Marketing, vol. 61, pp. 61-62.

- (1999): «Industria de los estudios de mercado en España 1998», Investigación y Marketing, vol. 64, pp. 74-76.

- (2001): «Industria de los estudios de mercado en España 2000», Investigación y Marketing, vol. 72, pp. $72-74$.

BOSCH, J. L., y TORRENTE, D. (1993): Encuestas Telefónicas y por Correo, Madrid: Centro de Investigaciones Sociológicas.

BRICK, J. M.; WAKSBERG, J.; KULP, D., y STARER, A. (1994): «Bias in list-assisted telephone samples», Public Opinion Quarterly, vol. 59, n. ${ }^{\circ}$ 2, pp. 218-235.

CENTRO DE INVESTIGACIONES SOCIOLÓGICAS (1998): Los comportamientos económicos de los españoles: ahorro y consumo, Madrid: Centro de Investigaciones Sociológicas, estudio n. ${ }^{\circ} 2287$, realizado en octubre.

COLLINS, M. (1999): «Sampling for UK telephone surveys», Journal of the Royal Statistical Society, Series A, vol. 162, part 1, pp. 1-4.

DE LEEUW, E., et al. (2002): «Have Telephone Surveys a Future in the 21-th century?», en International Conference on Improving Survey, 25-28 de agosto de 2002, Universidad de Copenhague, Dinamarca.

DeMAIO, T. (1980): «Refusals: Who, Where and Why», Public Opinion Quarterly, vol. 44, pp. 222-233.

DE MIGUEL, A. (1994): La sociedad española 1993-94, Madrid: Alianza.

DÍAZ DE RADA, V. (2000): «Modos de entrar en relación para la toma de datos: entrevista personal, telefónica y postal», en J. Martínez Gastey et al., La Investigación en Marketing, Barcelona: AEDEMO, pp. 424-443.

- (2004): Estrategias de Consumo y Estilos de Vida en la sociedad navarra del siglo xxı, Pamplona: Universidad Pública de Navarra.

DILLMAN, D. A. (1978): Mail and Telephone Surveys, New York: Wiley.

ELZO, J. (1992): Euskalherría en la encuesta europea de valores: ¿son los vascos diferentes?, Bilbao: Universidad de Deusto.

FOREMAN, J., y COLLINS, M. (1991): "The viability of random digit dialling in the UK», Journal of the Market Research Society, vol. 33, pp. 219-227.

GOBIERNO DE NAVARRA (1997): Estadística de la población de Navarra 1996. Principales resultados, Pamplona: Gobierno de Navarra, Departamento de Economía y Hacienda.

- (1998): Estadística de la población de Navarra 1996. Población según los estudios cursados, Pamplona: Gobierno de Navarra, Departamento de Economía y Hacienda.

GOYDER, J. (1987): The Silent Minority, Cambridge: Polity Press. 
GROVES, R. M. (1989): Survey error and survey cost, New York: Wiley.

HAGEN, D. E., y COLLIER, C. M. (1983): «Must respondent selection procedures for telephone surveys be invasive? », Public Opinion Quarterly, vol. 47, pp. 547-556.

INSTITUTO DE ESTADíSTICA DE NAVARRA (2004): Movimiento natural de la población, Pamplona: Gobierno de Navarra, Departamento de Economía y Hacienda. http:cfnavarra. es/estadística

INSTITUTO NACIONAL DE ESTADÍSTICA (INE) (2004): España al comienzo del siglo xxı. Censos de Población y Viviendas 2001, Madrid: INE.

KEETER, S. (1995): «Estimating telephone noncoverage bias with a telephone survey», Public Opinion Quarterly, vol. 59, pp. 196-217.

KISH, L. (1949): «A procedure for objective respondent selection within the household», Journal of the American Statistical Association, vol. 44, pp. 92-115.

KUUSELA, V., y SIMPANEN, M. (2002): «Effects of Mobile Phones on Telephone Survey», en International Conference on Improving Survey, 25-28 de agosto de 2002, Universidad de Copenhague, Dinamarca.

KVIZ, F. J. (1984): «Bias in a Directory Sample for a Mail Survey of Rural Household», Public Opinion Quarterly, vol. 48 , pp. 801-806.

LAVRAKAS, P. J. (1993): Telephone Survey Methods, Newbury Park-California: Sage Publicatons.

LEPKOWSKI, J. M. (1988): «Telephone survey sampling methods in the United States», en R. M. Groves et al., Telephone Survey Methodoly, New York: Wiley, pp. 73-98.

LESSLER, J. T., y KALSBEEK, W. D. (1992): Nonsampling Error in Surveys, New York: Wiley.

LÓPEZ BLASCO, A.; HERNÁNDEZ ARISTU, J., y VISCARRET, J. J. (2002): Informe Juventud en Navarra 2000, Pamplona: Gobierno de Navarra.

McDANIEL, R. W., et al. (1987): «Do Topic Differences Affect Survey Non-Response?», Journal of the Market Research Sociey, vol. 22, pp. 55-66.

NEDERHOF, A. J. (1988): «Effects of a Final Telephone Reminder and Questionnaire Cover Design in Mail Surveys», Social Science Research, vol. 17, pp. 353-361.

NOBLE, I.; MOON, N., y McWEY, D. (1998): «Bringing it all back home», Journal Proceedings Conference Market Research Society, pp. 35-53.

PASADAS DEL AMO, S.; ZARCO URIBE-ECHEVARRÍA, M., y SORIA ZAMBRANO, M. (2004): La telefonía móvil: un nuevo reto para las encuestas telefónicas, Córdoba: Documento de trabajo del Instituto de Estudios Sociales de Andalucía, Consejo Superior de Investigaciones Científicas, IESA 15-04.

RUIZ OLABUÉNAGA, J. I. (1998): La juventud liberta, Bilbao: Banco Bilbao Vizcaya.

SUDMAN, S. (1973): «The uses of telephone directories for survey sampling», Journal of Marketing Research, vol. 10, pp. 204-207.

THOMAS, R., y PURDON, S. (1994): «Telephone Methods for Social Surveys», Social Research Update, vol. 8, pp. 1-6.

TRAUGOTT, M. W., et al. (1987): «Using Dual Frame Designs to Reduce Nonresponse in Telephone Surveys», Public Opinion Quarterly, vol. 51, pp. 522-539.

TUCKER, C.; LEPKOWSKI, J. M.; CASADY, R. J., y GROVES, R. M. (1992): «Commercial residential telephone lists: their characteristics and uses in survey design», Social Science Computer Review, vol. 10, pp. 158-172.

WERT, J. I. (1994): «La Encuesta Telefónica», en J. Ibáñez, F. Alvira y M. García Ferrando, El Análisis de la Realidad Social, Madrid: Alianza Universidad, pp. 177-188. 


\section{ANEXO 1: CUESTIONARIO UTILIZADO}

¿SON DISTINTOS LOS HÁBITOS DE CONSUMO DE LOS NAVARROS?

Un estudio sobre hábitos y actitudes de consumo de la población navarra

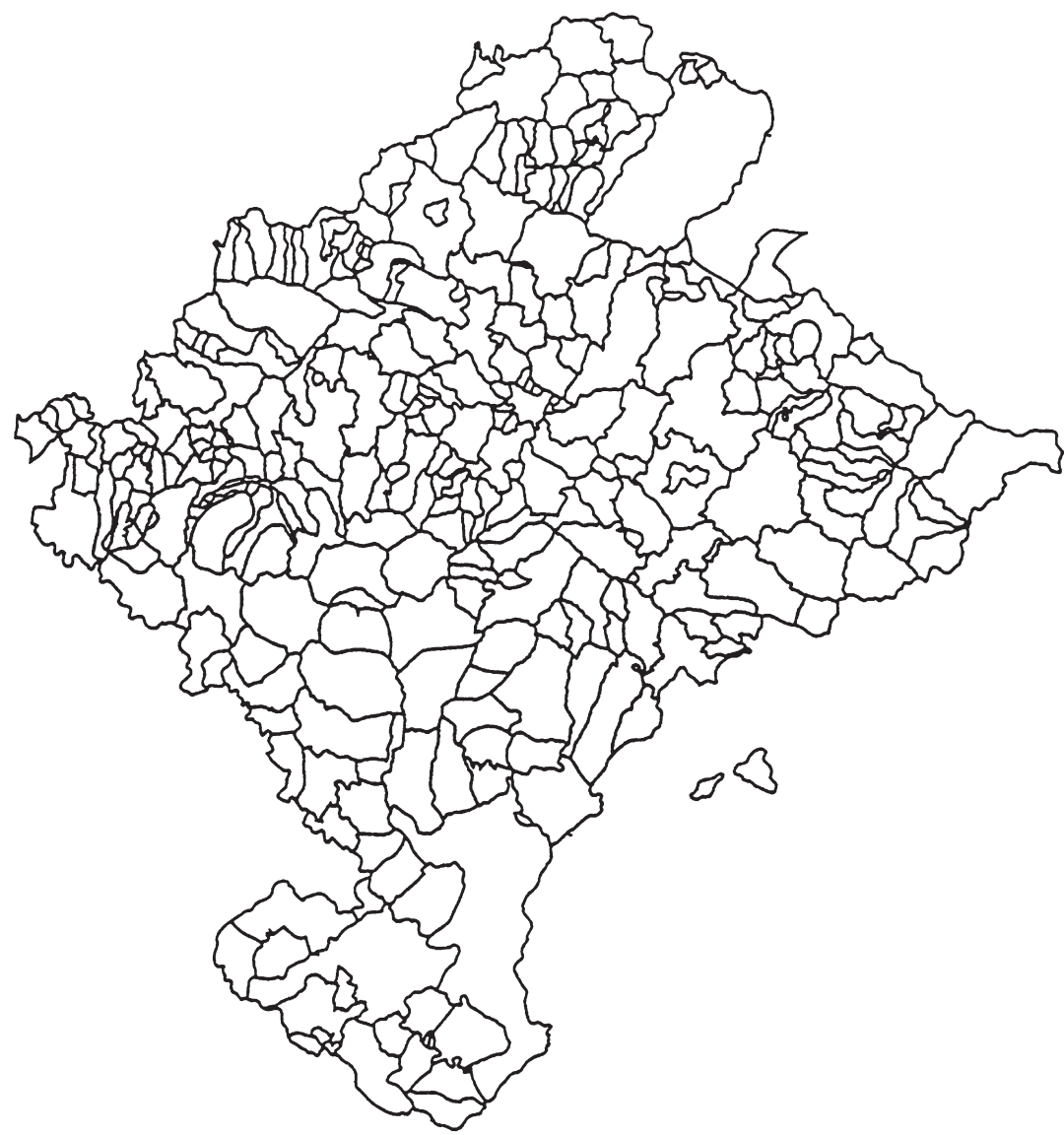

Muchísimas gracias por su colaboración.

Por favor, responda a todas las preguntas.

Puede utilizar el espacio de la parte de atrás de este cuaderno para cualquier comentario. Cuando esté terminado introdúzcalo en el sobre adjunto y envíelo a:

Universidad Pública de Navarra

Departamento de Sociología

Apartado n. ${ }^{\circ} 52$ F.D.

31080 Pamplona

Tfno.: 16-89-03 
01. ¿Cuánto tiempo pasa usted a la semana viendo escaparates? (Marcar una respuesta)

- CASI TODOS LOS DÍAS ME DETENGo A VER ESCAPARATES, YA QUE ASÍ VEO LO QUE NECESITO.

- DE VEZ EN CUANDO, CUANDO VEO ALGO QUE ME LLAMA LA ATENCIÓN, PERO SON POCAS VECES.

- SOLAMENTE ME PREOCUPO DE VER ESCAPARATES CUANDO VOY A COMPRAR ALGO, O CUANDO NECESITO ALGUNA COSA.

- NUNCA ME DETENGO A VER ESCAPARATES.

02. ¿Le gustan las rebajas? (Marcar una respuesta)

- Sí ME GUSTAN, PORQUE ENCUENTRO PRODUCTOS QUE NECESITABA A MUY BUEN PRECIO.

- MUY POCO. CASI NUNCA HAY PRODUCTOS REALMENTE ÚTILES.

- NO ME GUSTAN, PUESTO QUE SIEMPRE ACABO COMPRANDO PRODUCTOS QUE NO NECESITO.

03. ¿Cómo cree usted que son los productos que se venden en rebajas? (Marcar una respuesta)

- HAY PRODUCTOS QUE SON VERDADERAS «GANGAS», SÓLO ES NECESARIO SABER ENCONTRARLOS.

- SUELEN SER PRODUCTOS DE CALIDAD, REBAJADOS DE PRECIO.

- SON PRODUCTOS UN POCO PASADOS DE MODA, PERO QUE ESTÁN MUY BIEN.

- LA MAYOR PARTE DE LOS PRODUCTOS SON AUTÉNTICOS ENGAÑOS, LAS REBAJAS SON RECLAMOS PARA VENDER LOS PRODUCTOS DE BAJA CALIDAD.

04. ¿Suele comprar productos que están de oferta o rebajados? (Marcar una respuesta)

- SIEMPRE.

- CASI SIEMPRE.

- SÓLO EN TEMPORADA DE REBAJAS (REBAJAS DE ENERO, ETC.).

- NUNCA O CASI NUNCA. 
05. ¿Que cree usted que es mejor, tener menos ropa pero que sea de buena calidad, o más ropa, aunque no sea de tanta calidad? (Marcar una respuesta)

- MENOS ROPA DE MÁS CALIDAD.

- MÁS ROPA AUNQUE NO SEA DE TANTA CALIDAD.

06. ¿Le gusta a usted que en la ropa que lleva se vea la marca? (Marcar una respuesta)

- sí.

- INDIFERENTE.

- NO.

07. ¿Por qué cree usted que se pone de moda una determinada marca? (Marcar una respuesta)

- PORQUE LO UTILIZA UNA DETERMINADA GENTE.

- CALIDAD.

- FUNCIONALIDAD, COMODIDAD.

- NOVEDAD, INNOVACIÓN.

- PUBLICIDAD.

08. ¿Cuando deja de usar prendas, suele ser porque están ya en mal estado, porque ya no le gustan o porque no están de moda? (Marcar una respuesta)

- MAL ESTADO.

- NO ME GUSTAN YA.

- SE HAN PASADO DE MODA.

09. ¿Suele comprar cosas que luego no usa? (Marcar una respuesta)

- sí, FRECUENTEMENTE.

- ALGUNA VEZ QUE OTRA.

- NO, NUNCA. 
10. ¿Podría decirme si está de acuerdo o en desacuerdo con cada una de estas acciones?

(Para cada acción, marcar si se está de acuerdo o en desacuerdo).

Si tuviera dinero, compraría más productos de lujo, simplemente por el placer que produce el lujo en sí mismo.

- ACUERDO.

- DESACUERDO.

Me he encontrado en ciertas ocasiones con que había gastado más de lo que realmente tenía.

- ACUERDO.

- DESACUERDO.

Me encanta ir de compras. Para mí comprar es un placer.

- ACUERDO.

- DESACUERDO.

En lo que se refiere a las marcas soy fiel. Tengo mis costumbres.

- ACUERDO.

- DESACUERDO.

Disfruto mucho comprando cosas que no tenía previsto.

- ACUERDO.

- DESACUERDO.

Cuando compro, siempre estoy abierto a los cambios y a las nuevas ideas.

- ACUERDO.

- DESACUERDO.

De vez en cuando me gusta comprar o llevar cosas que sé que compra o lleva gente que piensa como yo.

- ACUERDO.

- DESACUERDO.

Estoy dispuesto a invertir mucho esfuerzo para encontrar la marca que me conviene.

- ACUERDO.

- DESACUERDO. 
11. De la siguiente lista de cualidades que se pueden transmitir a los niños en el hogar. ¿cuáles cree usted que son las CUATRO más importantes? (Marcar las CUATRO más importantes)

- BUENOS MODALES.

- INDEPENDENCIA.

- ESFUERZO EN EL TRABAJO.

- SENTIDO DE LA RESPONSABILIDAD.

- IMAGINACIÓN.

- TOLERANCIA Y RESPETO HACIA LOS DEMÁS.

- SOBRIEDAD Y ESPÍRITU DE AHORRO.

- DETERMINACIÓN, PERSEVERANCIA.

- FE RELIGIOSA.

- ESPÍRITU DE SACRIFICIO.

- OBEDIENCIA.

- HONRADEZ.

12. ¿Qué ideal de vida prefiere, el de «trabajar, ahorrar y subir» o el de «trabajar, consumir y disfrutar»? (Marcar una respuesta)

- TRABAJAR, AHORRAR Y SUBIR.

- LOS DOS SON PARECIDOS.

- NINGUNO DE LOS DOS.

- TRABAJAR, CONSUMIR Y DISFRUTAR.

13. ¿Le gustaría a usted ganar más dinero, aunque ello supusiera más preocupaciones? (Marcar una respuesta)

- sí.

- NO.

14. ¿Cree usted que la gente que le rodea se mueve fundamentalmente por dinero? (Marcar una respuesta)

- sí.

- NO. 
15. ¿Podría decirme su grado de acuerdo con cada una de las siguientes frases? (Para cada acción, marcar si se está de acuerdo o en desacuerdo)

RESPUESTAS

Es fácil llegar a ganar mucho dinero en poco tiempo si se tienen pocos escrúpulos:

Los que tienen mucho dinero es fundamentalmente porque han tenido mucha suerte

El dinero ayuda a la felicidad

Cada año que pasa, es más alto el nivel de corrupción de los políticos que están en el poder
Muy de Bastante de Poco de Nada de acuerdo acuerdo acuerdo acuerdo Muy de Bastante de Poco de Nada de acuerdo acuerdo acuerdo acuerdo Muy de Bastante de Poco de Nada de acuerdo acuerdo acuerdo acuerdo Muy de Bastante de Poco de Nada de acuerdo acuerdo acuerdo acuerdo

16. ¿Cuál es su nivel de estudios? Indicar los estudios acabados de mayor nivel) (Marcar una respuesta)

- MENOS DE ESTUDIOS PRIMARIOS.

- ESTUDIOS PRIMARIOS (SABER LEER Y ESCRIBIR, CERTIFICADO DE ESCOLARIDAD).

- FORMACIÓN PROFESIONAL.

- BACHILLER ELEMENTAL.

- BACHILLER SUPERIOR, B.U.P., R.E.M.

- ESTUdIOS DE GRADO MEDIO (ESCUELA UNIVERSITARIA).

- UNIVERSITARIOS O TÉCNICOS DE GRADO SUPERIOR.

17. ¿Cuántas personas viven ahora en su hogar

(Apuntar número)

18. ¿Podría decirme qué edad tiene Ud.?

19. Señale si es hombre o mujer (Marcar una respuesta)

- HOMBRE.

- MUJER. 
Si hay algo que a usted le gustaría añadir acerca de sus hábitos de consumo o cualquier otro comentario sobre los temas tratados en este cuestionario, hágalo en el espacio que sigue a continuación.

Agradecemos su ayuda y esfuerzo al responder a este cuestionario. Si le gustaría obtener un resumen de los resultados de este estudio, por favor, ponga su nombre y dirección en la solapa del sobre de respuesta (no en este cuestionario). Se lo enviaremos con mucho gusto. 


\section{ANEXO 2: CARTA DE PRESENTACIÓN QUE ACOMPAÑA AL CUESTIONARIO}

Pamplona, 20 de abril de 1998

Nombre y Apellidos del destinatario

Dirección (Calle y número)

Municipio y código postal

\section{Estimado Sr. Apellidos:}

En los últimos años se están realizando un gran número de investigaciones sobre los hábitos y actitudes de compra de los residentes en diferentes núcleos de población. Como le indicamos en la carta anterior, nos gustaría contar con su ayuda, puesto que el conocimiento de estos hábitos puede proporcionarnos notables mejoras a todos los ciudadanos al aproximar la oferta existente a las necesidades de todos nosotros. Investigaciones similares a ésta se están realizado en otras zonas, y consideramos que la comparación de los hábitos de los navarros con los de otras áreas va a contribuir notablemente a conocernos mejor.

Como ya le indicamos la semana pasada, su hogar forma parte de un pequeño número de personas que están siendo preguntadas para conocer su opinión sobre estos asuntos. Para ello se ha realizado una selección entre todos los hogares de Navarra. Para que los resultados representen verdaderamente lo que piensa la población navarra es importante que cada cuestionario sea rellenado y reenviado a la Universidad Pública de Navarra. Así, nos gustaría que este cuestionario que le enviamos sea respondido por la mayor de sus hijas. Si no tiene hijas debería ser respondido por el mayor de sus hijos, o por usted mismo.

Puede estar seguro que sus respuestas serán analizadas de forma confidencial y se utilizarán únicamente con fines científicos. Como ha podido observar, el cuestionario tiene un número de identificación en su parte superior derecha que se ha colocado únicamente como control de la correspondencia. Deseamos insistir en que su nombre NUNCA estará unido al cuestionario ni a las respuestas del mismo. El número se destruye cuando el cuestionario llega a la Universidad Pública de Navarra.

Tenga por seguro que los resultados obtenidos servirán para conocer mejor nuestros hábitos y gustos de compra. También se publicarán en revistas especializadas. Si lo desea podemos enviarle un resumen de los resultados obtenidos escribiendo «deseo copia de resultados» en la parte de atrás del sobre de respuesta, escribiendo después su nombre y 
dirección. Por favor, no escriba esta información en el cuestionario. Hágalo únicamente en el sobre de respuesta.

Estaremos a su disposición para responder las cuestiones que usted pueda plantear. Por favor, no dude en comunicarse con nosotros si tiene alguna pregunta.

Gracias por su colaboración en este importante proyecto.

Atentamente:

Vidal Díaz de Rada

Director del Proyecto 
When the target population of a survey comprises specialist collectives (doctors, lawyers, etc.) it is common to use the professional directories of each individual collective, but what happens when the study target is the population at large? This paper provides an answer to this question, analysing up to what extent it is correct to use the telephone directory as a population framework for personal interviews (face to face), telephone surveys or those done by mail. We show the results of a research study carried out with a postal survey, which was subsequently completed with a telephonic and then a personal survey. The hypothesis proposed claims that the main problem about using the telephone directory as a population framework is the difficulty in obtaining an appropriate level of representativeness of the reality that is the object of study.

Key words: Postal Surveys, Telephone Surveys, Population Framework, No Coverage. 